\title{
CIENCIAMATRIA
}

Revista Interdisciplinaria de Humanidades, Educación, Ciencia y Tecnología

Año VII. Vol. VII. N¹2. Enero - Junio. 2021

Hecho el depósito de ley: pp201602FA4721

ISSN-L: 2542-3029; ISSN: 2610-802X

Universidad Nacional Experimental Francisco de Miranda (UNEFM). Santa Ana de Coro. Venezuela

Rosa del Rocío Pinargote-Chancay; Derlin Alicia Delgado-Janumis; Maria Elena Pincay-Cañarte; Lorena Pilar Lino-Solis

DOI 10.35381/cm.v7i12.455

\section{Patrón de Conocimiento Sociopolítico: Disciplina y práctica de la profesión en Enfermería}

\section{Pattern of Socio-Political Knowledge: Discipline and practice of the Nursing profession}

\author{
Rosa del Rocío Pinargote-Chancay \\ rosa.pinargote@unesum.edu.ec \\ Universidad Estatal del Sur de Manabí, Jipijapa \\ Ecuador \\ https://orcid.org/0000-0001-9899-9243 \\ Derlin Alicia Delgado-Janumis \\ derlindelgado.21@hotmail.com \\ Universidad Estatal del Sur de Manabí, Jipijapa \\ Ecuador \\ https://orcid.org/0000-0002-8311-6740 \\ Maria Elena Pincay-Cañarte \\ maria.pincay@unesum.edu.ec \\ Universidad Estatal del Sur de Manabí, Jipijapa \\ Ecuador \\ https://orcid.org/0000-0003-4110-9275 \\ Lorena Pilar Lino-Solis \\ loreli2008@hotmail.com \\ Centro Clínico Quirúrgico Hospital del Día Norte Tarqui, Guayaquil \\ Ecuador \\ https://orcid.org/0000-0001-5012-8887
}

Recibido: 15 de enero de 2021

Aprobado: 15 de marzo de 2021 
CIENCIAMATRIA

Revista Interdisciplinaria de Humanidades, Educación, Ciencia y Tecnología

Año VII. Vol. VII. N¹2. Enero - Junio. 2021

Hecho el depósito de ley: pp201602FA4721

ISSN-L: 2542-3029; ISSN: 2610-802X

Universidad Nacional Experimental Francisco de Miranda (UNEFM). Santa Ana de Coro. Venezuela

Rosa del Rocío Pinargote-Chancay; Derlin Alicia Delgado-Janumis; Maria Elena Pincay-Cañarte;

Lorena Pilar Lino-Solis

\title{
RESUMEN
}

El objetivo de la investigación se basó en analizar los patrones de conocimiento sociopolítico asociados a la disciplina y práctica de la profesión en enfermería. Basado en una metodología de corte bibliográfico - documental; donde la hermenéutica se constituyó en la principal fuente de generación de información y con un diseño fue noexperimental. El corpus del estudio lo constituyeron las lecturas relacionadas a teorías en enfermería, contenidas en diferentes textos, artículos científicos y de opinión disponibles en la web. Se pudo evidenciar transversalidad en el patrón de conocimiento socio-político, dado que permite conocer y reconocer el contexto desde una perspectiva holística, permitiendo trasformar sus políticas y leyes en materia legislativa propias de la salud y el ejercicio de la profesión de la enfermería.

Descriptores: Política de la salud; lucha contra las enfermedades; servicio de salud. (Palabras tomadas del Tesauro UNESCO).

\begin{abstract}
ABSTRAC
The objective of the research was based on analyzing the socio-political knowledge patterns associated with the discipline and practice of the nursing profession. Based on a bibliographic-documentary methodology; where hermeneutics became the main source of information generation and with a non-experimental design. The corpus of the study was made up of readings related to nursing theories, contained in different texts, scientific articles and opinion articles available on the web. It was possible to show transversality in the pattern of socio-political knowledge, since it allows knowing and recognizing the context from a holistic perspective, allowing the transformation of its policies and laws in legislative matters related to health and the practice of the nursing profession.
\end{abstract}

Descriptors: Health policy; disease control; health services. (Words taken from the UNESCO Thesaurus). 


\section{CIENCIAMATRIA}

Revista Interdisciplinaria de Humanidades, Educación, Ciencia y Tecnología

Año VII. Vol. VII. N¹2. Enero - Junio. 2021

Hecho el depósito de ley: pp201602FA4721

ISSN-L: 2542-3029; ISSN: 2610-802X

Universidad Nacional Experimental Francisco de Miranda (UNEFM). Santa Ana de Coro. Venezuela

Rosa del Rocío Pinargote-Chancay; Derlin Alicia Delgado-Janumis; Maria Elena Pincay-Cañarte; Lorena Pilar Lino-Solis

\section{INTRODUCCION}

Dar respuesta al problema sociopolítico desde la enfermería, es y debe ser considerada de vital importancia por todas las personas, en tanto que la realidad social y política de la sociedad viene a estar caracterizada en problemas como: exclusión social, pérdida de credibilidad y confianza hacia sus gobernantes, desigualdad de ingresos y riqueza, dependencia económica, fragmentación y desarticulación de la sociedad, fluctuación e inestabilidad en los procesos sociales, debilidad para con el sistema de justicia, seguridad y defensa, creciente corrupción administrativa, impunidad generalizada, prácticas clientelistas y múltiples violencias, entre otras, (Ramírez-Elizondo, et al., 2013).

En atención a ello, se puede indicar que son las formas de dar respuesta a diversas preguntas y problemas que surgen en esta disciplina, que desde la investigación se determina el tipo de conocimiento, independientemente de las metas que la disciplina y profesión requieren para desarrollarse, conducir la organización y aplicar el conocimiento (Escobar-Castellanos \& Sanhueza-Alvarado, 2018).

Por otra parte, hablar de estos en la profesión implica referencial, tal como lo expone Barbara Carper, que existen varios patrones vitales para el ejercicio de la enfermería; el empírico que hace referencia a la ciencia de enfermería; el estético; el personal; y el ético permite el desarrollo y práctica de la moral en enfermería (Carper, 1978).

Así mismo, están entendidos como un desafío para la enfermería, porque es una labor que tiende a ser invisible socialmente, solo al interior del gremio se reconoce su capacidad para actuar en tiempos de dolor y sufrimiento. De allí que, la enfermería es una labor con merecido reconocimiento por su contribución a la construcción, decisión y ejecución de diversas líneas de cuidado dentro de la dinámica de la sociedad (Persegona, et al., 2009).

En tal sentido, el desarrollo del conocimiento y la práctica son elementos indispensables de fundamentación de la profesión; dado que se expresan de modo integrado, develando entre sí, conexiones que permiten la expresión del cuidado y de las intervenciones de 


\section{CIENCIAMATRIA}

Revista Interdisciplinaria de Humanidades, Educación, Ciencia y Tecnología

Año VII. Vol. VII. N¹2. Enero - Junio. 2021

Hecho el depósito de ley: pp201602FA4721

ISSN-L: 2542-3029; ISSN: 2610-802X

Universidad Nacional Experimental Francisco de Miranda (UNEFM). Santa Ana de Coro. Venezuela

Rosa del Rocío Pinargote-Chancay; Derlin Alicia Delgado-Janumis; Maria Elena Pincay-Cañarte; Lorena Pilar Lino-Solis

enfermería de forma holística hacia el paciente y la sociedad. El presente trabajo tiene como objetivo analizar los patrones de conocimiento sociopolítico asociados a la disciplina y práctica de la profesión en enfermería.

\section{Recorrido teórico}

Para entender qué es enfermería y la naturaleza de sus fundamentos filosóficos, se requiere un amplio ámbito de conocimiento que procede de las perspectivas humanísticas y científicas (White, 1995). El cuerpo de saberes que sirve de fundamento para la práctica tiene patrones, formas y estructuras que dan expectativas a los horizontes de la disciplina, entenderlos es esencial para la enseñanza y el aprendizaje de enfermería, este entendimiento no proyecta la amplitud del conocimiento, pero llama la atención sobre la cuestión relativa al significado de conocer y sobre las clases de conocimiento que se pueden determinar y que aportan de manera, más significativa, al conocimiento de enfermería (Velandia-Mora, 1998).

\section{Patrón del conocimiento sociopolítico en enfermería}

El patrón de conocimiento socio-político (Osorio-Castaño, 2016), hace parte del conocimiento empírico, afirmación que puede llevar a diluir y a limitar su desarrollo, expansión y aplicación, admitiendo el conocimiento del contexto de la enfermera y el paciente y su contexto socio-político como la práctica profesional que incluye el entendimiento de la sociedad y sus políticas en enfermería. De allí que un patrón de conocimiento puede hacer alusión a los aspectos asociados a la percepción, observación, experiencia y comprensión que se repiten y reconocen con anticipación mediante señales cuales se convierten en una referencia que permite aprehender y comunicar un objeto para favorecer la acción (Chinn \& Kramer, 2011). La comprensión, sobre todo en los empleados de enfermería, es esencial para su enseñanza y aprendizaje, lo cual permite identificar la variabilidad de aspectos que competen a esta 


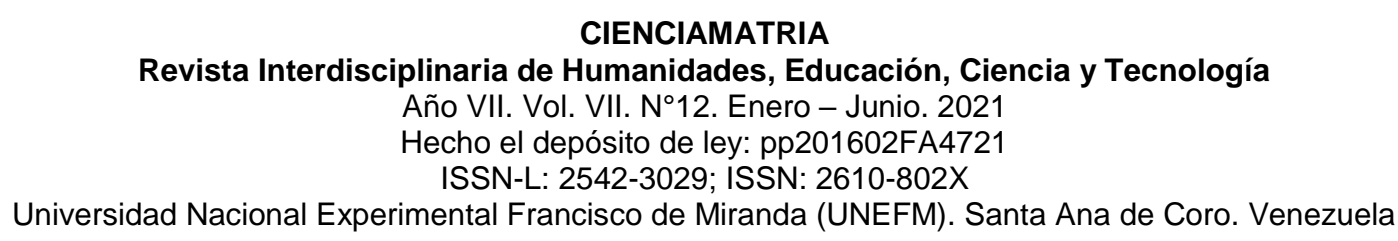

Rosa del Rocío Pinargote-Chancay; Derlin Alicia Delgado-Janumis; Maria Elena Pincay-Cañarte; Lorena Pilar Lino-Solis

disciplina y qué clase de conocimientos son de mayor valor en la misma (EscobarCastellanos \& Sanhueza-Alvarado, 2018).

Cada patrón cuando reconoce que los cambios son inherentes al desarrollo del conocimiento de la profesión (Carper, 1978), les proponen orientaciones que permiten orientar los problemas y las preguntas de la disciplina, de tal forma que aumentan su complejidad y su diversidad epistemológica. Esta autora propone que el conocimiento de enfermería puede ser organizado a partir de lo personal, estético, ético y moral. Posteriormente, se adiciona (Velandia-Mora, 1998), el patrón político social, y se propone el patrón emancipatorio (Osorio-Castaño, 2016).

Estos patrones en la enfermería son elementos indispensables de fundamentación para el desarrollo de la práctica; se expresan de manera integrada, existiendo entre ellos conexiones que permiten la expresión del cuidado y de las intervenciones de enfermería de forma holística (Escobar-Castellanos \& Sanhueza-Alvarado, 2018). Asimismo, el patrón de conocimiento socio-político implica el conocimiento del contexto socio-político (Osorio-Castaño, 2016), el paciente y la enfermería como práctica profesional, incluyendo el entendimiento de la sociedad y sus políticas

Esto permite entender la importancia de enfermería en la sociedad y viceversa, del rol de abogacía que ejerce, sin dejar de lado las políticas establecidas socialmente, dando respuesta a la situación social donde la enfermera establece relaciones con el paciente, abordando el contexto donde tiene lugar el cuidado (Escobar-Castellanos \& SanhuezaAlvarado, 2018).

Por consiguiente, la profesión de enfermería se interesa en las actividades que desarrollan y canalizan sus practicantes para lograr la promoción y el mejoramiento de la salud y el bienestar de los seres humanos (White, 1995). Desde la perspectiva de la disciplina, el cuerpo de conocimientos y su experiencia en la interacción con las necesidades del ser humano, permiten orientar sus esfuerzos a desarrollar una profesión con posiciones críticas hacia lo social. 


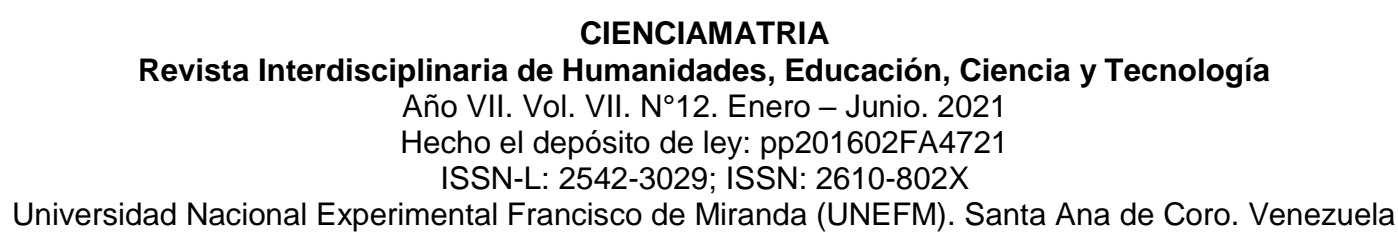

Rosa del Rocío Pinargote-Chancay; Derlin Alicia Delgado-Janumis; Maria Elena Pincay-Cañarte; Lorena Pilar Lino-Solis

Una parte primordial, que muestra el avance de la disciplina respecto al patrón del conocimiento socio-político es el desarrollo del pensamiento crítico y reflexivo respecto a los contextos donde se ejerce el cuidado. El reconocer que la persona es un ser activo y holístico que interactúa con el ambiente, ayuda a que el conocimiento de enfermería salga de la perspectiva biomédica bajo la cual ha funcionado por mucho tiempo (MedinaMoya, 2017), y aquí, en este reconocimiento de necesidades y vulnerabilidades del ser humano, es que se hace evidente que la profesión ha mejorado de manera continua desde el ser y el hacer (Ramírez-Elizondo, et al., 2013).

\section{METODOLOGIA}

Se utilizó una metodología de corte bibliográfico - documental (Balestrini, 2011), la cual consistió en la recolección de la información que se encuentra vinculada a la delimitación de un diseño bibliográfico a partir de la consulta de libros y documentos de internet, los cuales sirvieron de apoyo y respaldo al estudio planteado. Todo ello, bajo un enfoque hermenéutico de las teorías relacionadas con los patrones de conocimiento sociopolítico en enfermería. La hermenéutica se constituyó en la principal fuente de generación de información, que es el arte de explicar textos o escritos, considerada como la vía hipotética deductiva más efectiva. El corpus del estudio lo constituyeron las lecturas relacionadas a dichas teorías en enfermería (Sandin, 2003).

El diseño fue no-experimental por cuanto no se manipuló deliberadamente la variable patrones de conocimiento socio-político en enfermería. De acuerdo con su intención, la misma asumió una postura epistemológica basada en el idealismo-deductivo, en tanto que se partió del conocimiento previo de la variable para presentar resultados hermenéuticos de carácter objetivo acerca de sus teorías. 


\section{CIENCIAMATRIA}

Revista Interdisciplinaria de Humanidades, Educación, Ciencia y Tecnología

Año VII. Vol. VII. N¹2. Enero - Junio. 2021

Hecho el depósito de ley: pp201602FA4721

ISSN-L: 2542-3029; ISSN: 2610-802X

Universidad Nacional Experimental Francisco de Miranda (UNEFM). Santa Ana de Coro. Venezuela

Rosa del Rocío Pinargote-Chancay; Derlin Alicia Delgado-Janumis; Maria Elena Pincay-Cañarte; Lorena Pilar Lino-Solis

\section{ANÁLISIS Y DISCUSIÓN}

Posterior a las revisiones bibliográficas de los documentos recopilados y el análisis hermenéutico del mismo, fue posible constatar que:

El patrón de conocimiento socio-político como elemento del conocimiento práctico (Persegona, et al., 2009), permite disolver y/o restringir su progreso, expansión y aplicación. Sin embargo, se señala como práctica profesional incluyendo el entendimiento de la sociedad de enfermería y sus políticas. Este patrón no se considera tan inmediato en la relación enfermera - paciente, en tanto va más allá de su espacio institucional (White, 1995).

El profesional de enfermería como miembro del equipo de salud tiene gran responsabilidad social con estas personas, en especial a lo que compete a la educación para la salud, el acceso a servicios y el autocuidado, donde la relevancia social de la profesión permite establecer planes de intervención social, a través de la comunidad para la definición de sus problemas y búsqueda de soluciones, para disminuir el impacto en las enfermedades. Todo ello, entendiendo que la exposición de la sociedad a malas condiciones de salud, desnutrición, riesgos medioambientales y psicosociales, va más allá de los problemas económicos (Mata-Cortés, 2002).

Durante el ejercicio de la enfermería, se ha de defender el derecho de las personas al cuidado individual, según su propio "juicio clínico y sus preferencias", con una visión de justicia distributiva y con equidad En tanto que, cuando se encuentra con un posicionamiento político, pero que incluye a la ciencia entre sus prioridades, consigue optar y opinar acerca de cuestiones que interfieren directamente en la producción de salud, movilizando a pacientes y colegas a convertirse en corresponsables por el cuidado, alcanzando una posible obtención de autonomía y emancipación profesional (Lopera-Betancur, 2015).

Es posible que el profesional de la enfermería, como gestor del cuidado emprenda acciones de educación y orientación, implemente prácticas de autocuidado, y 


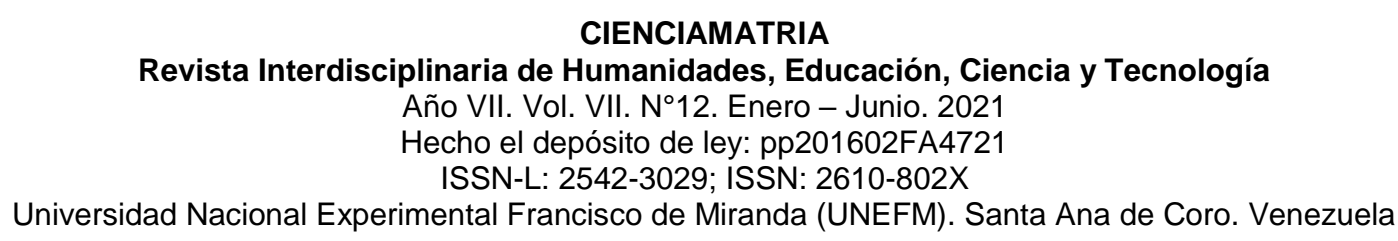

Rosa del Rocío Pinargote-Chancay; Derlin Alicia Delgado-Janumis; Maria Elena Pincay-Cañarte; Lorena Pilar Lino-Solis

comunicación asertiva dirigida a la persona, la familia y la comunidad, en pro de mitigar las dificultades que presentan los servicios de salud, así como para favorecer las medidas de protección personal y la abogacía en beneficio del ser humano (Rutz-Porto y Buss-Thofehrn, 2015).

Es importante reconocer que muchos puestos y responsabilidades del sector sanitario están ocupados por personal de la enfermería, pero es imperativo que se abran caminos en espacios de toma de decisiones, y en el diseño de las políticas de salud con el fin de ampliar el conocimiento y traer innovaciones y de esta manera evitar la imposición de otros profesionales es decir que se haga visible su profesión. En este sentido, se indica que el trabajar en equipo permitiría claros beneficios, no sólo para el ambiente de trabajo, sino también para los pacientes, que, de ser así, serán beneficiarios de una atención sanitaria de calidad y lograr construir colectivamente una profesión más influyente, comprometida y activa en las decisiones de políticas públicas, sociales e institucionales (Cassiani, et al., 2020).

\section{CONCLUSIONES}

El desarrollo del patrón socio político contribuye a la práctica cuando el profesional de enfermería aboga por el derecho a la salud de los sujetos de cuidado; aporta a la disciplina al contemplar el contexto como una oportunidad de visibilización y expresión a través de la participación junto con las que podrían considerarse sus características como la movilización, la comunicación y el liderazgo; en la medida que se realicen abordajes más precisos del significado de la participación en enfermería y su aporte a la profesión está en el trabajo para hacerse visible ante la sociedad.

Se puede evidenciar transversalidad en el patrón de conocimiento socio-político, dado que permite conocer y reconocer el contexto desde una perspectiva holística, permitiendo trasformar sus políticas y leyes en materia legislativa propias de la salud y 


\begin{abstract}
CIENCIAMATRIA
Revista Interdisciplinaria de Humanidades, Educación, Ciencia y Tecnología

Año VII. Vol. VII. N¹2. Enero - Junio. 2021

Hecho el depósito de ley: pp201602FA4721

ISSN-L: 2542-3029; ISSN: 2610-802X

Universidad Nacional Experimental Francisco de Miranda (UNEFM). Santa Ana de Coro. Venezuela
\end{abstract}

Rosa del Rocío Pinargote-Chancay; Derlin Alicia Delgado-Janumis; Maria Elena Pincay-Cañarte; Lorena Pilar Lino-Solis

el ejercicio de la profesión de la enfermería, lo que implica tomar en cuenta los ambientes donde se gestan las experiencias de salud y enfermedad de las personas.

Los patrones de conocimiento socio político se fundan considerando las perspectivas de la dinámica de cada una de las personas, por lo que no son de tipo abstracto sino tangible facilitando conocer, entender y hasta modificar los contextos del profesional de la enfermería y de las personas en general.

\title{
REFERENCIAS CONSULTADAS
}

Balestrini, M. (2011). Cómo se elabora el proyecto de investigación. Caracas, Consultores Asociados.

Carper B. (1978). Fundamental patterns of knowing in nursing. ANS. Advances in nursing science, 1(1), 13-23. https://doi.org/10.1097/00012272-197810000-00004

Cassiani, S., Munar-Jimenez, E., Umpiérrez-Ferreira, A., Peduzzi, M., y Leija-Hernández, C. (2020). La situación de la enfermería en el mundo y la Región de las Américas en tiempos de la pandemia de COVID-19. [The situation of nursing in the world and the Region of the Americas at the time of the COVID-19 pandemic] Revista Panamericana de Salud Pública, 44(1), 1-2. https://doi.org/10.26633/RPSP.2020.64

Chinn, P. \& Kramer, M. (2011). Nursing's fundamental patterns of knowing. In: Integrated theory and knowledge development in nursing. [8va ed]. Elsevier Mosby.

Escobar-Castellanos, B, \& Sanhueza-Alvarado, O. (2018). Patrones de conocimiento de Carper y expresión en el cuidado de enfermería: estudio de revisión [Carper's knowledge patterns and expression in nursing care: review study]. Enfermería: Cuidados Humanizados, 7(1), 27-42. https://dx.doi.org/10.22235/ech.v7i1.1540

Lopera-Betancur, M. (2015). Cuidados al final de la vida: una oportunidad para fortalecer el patrón emancipatorio de enfermería. Avances en Enfermería, 33(1), 124-132.

Mata-Cortés, M. (2002). Pobreza y salud: La enfermería ante este reto. Revista de Enfermería del Instituto Mexicano del Seguro Social, 10(2), 61-66. 


\section{CIENCIAMATRIA}

Revista Interdisciplinaria de Humanidades, Educación, Ciencia y Tecnología

Año VII. Vol. VII. N¹2. Enero - Junio. 2021

Hecho el depósito de ley: pp201602FA4721

ISSN-L: 2542-3029; ISSN: 2610-802X

Universidad Nacional Experimental Francisco de Miranda (UNEFM). Santa Ana de Coro. Venezuela

Rosa del Rocío Pinargote-Chancay; Derlin Alicia Delgado-Janumis; Maria Elena Pincay-Cañarte; Lorena Pilar Lino-Solis

Medina-Moya, J. L. (2017). La pedagogía del cuidado: saberes y prácticas en la formación universitaria en enfermería. Consejo de Enfermería de la Comunidad Valenciana. https://n9.cl/orp6

Osorio-Castaño, J. (2016). Patrón de conocimiento socio-político en enfermería: reflexiones conceptuales. Revista Cuidarte, 7(2), 13521357. https://doi.org/10.15649/cuidarte.v7i2.319

Persegona, K, Rocha, D, Lenardt, M, \& Zagonel, I. (2009). O conhecimento político na atuação do enfermeiro [The political knowing in the acting of the nurse]. Escola Anna Nery, 13(3), 645-650. https://doi.org/10.1590/S1414-81452009000300027

Ramírez-Elizondo, N, Quintana-Zavala, M, Sanhueza-Alvarado, O, \& Valenzuela-Suazo, S. (2013). El paradigma emancipatorio y su influencia sobre el desarrollo del conocimiento en Enfermería [The emancipatory paradigm and its influence on the development of nursing knowledge]. Enfermería Global, 12(30), 410-421.

Ramírez-Elizondo, N., Quintana-Zavala, M., Sanhueza-Alvarado, O., \& ValenzuelaSuazo, S. (2013). The emancipatory paradigm and its influence on the development of nursing knowledge. Enfermera Global, 12(2), 410-421.

Rutz-Porto, A., y Buss-Thofehrn, M. (2015). El empoderamiento político de los enfermeros en la práctica hospitalaria. [The political empowerment of nurses in hospital practice] Index de Enfermería, 24(1-2), 20-24. https://doi.org/10.4321/S1132-12962015000100005

Sandin, M. (2003). Investigación Cualitativa en Educación. Capítulo 3. Perspectivas Teórico - epistemológicas en la investigación educativa. Madrid, McGraw-Hill.

Velandia-Mora, A. L. (1998). Enfermería: desarrollo teórico e investigativo. María Mercedes Durán de Villalobos. Avances en Enfermería, 16(1-2), 101-103.

White J. (1995). Patterns of knowing: review, critique, and update. ANS. Advances in nursing science, 17(4), 73-86. https://doi.org/10.1097/00012272-199506000$\underline{00007}$

(C2021 por los autores. Este artículo es de acceso abierto y distribuido según los términos y condiciones de la licencia Creative Commons Atribución-NoComercial-Compartirlgual 4.0 Internacional (CC BY-NC-SA 4.0)

(https://creativecommons.org/licenses/by-nc-sa/4.0/ 